\title{
Time-Delay Estimation in Multiple-Input Single-Output Systems
}

\author{
Fatih Kocak and Sinan Gezici \\ Department of Electrical and Electronics Engineering \\ Bilkent University \\ Bilkent, Ankara 06800, Turkey \\ E-mails: \{fkocak, gezici\}@ee.bilkent.edu.tr
}

\begin{abstract}
In this paper, the time-delay estimation problem is studied for multiple-input single-output (MISO) systems. First, a theoretical analysis is carried out by deriving the CramerRao lower bound (CRLB) for time-delay estimation in a MISO system. Then, the maximum likelihood (ML) estimator for the time-delay parameter is obtained, which results in a complex optimization problem in general. In order to provide a solution of the ML estimator with low computational complexity, ML estimation based on a genetic global optimization algorithm, namely, differential evolution (DE), is proposed. Simulation studies for various fading scenarios are performed to investigate the performance of the proposed algorithm.

Index Terms - Time-delay estimation, Cramer-Rao lower bound (CRLB), multiple-input single-output (MISO), differential evolution (DE), maximum likelihood (ML) estimator.
\end{abstract}

\section{INTRODUCTION}

Facilitating wireless networks in positioning applications besides the communications applications has been getting a growing attention recently. There are a lot of application areas and services that make use of positioning techniques. The typical examples for outdoor systems are enhanced 911 (E911), improved fraud detection, cellular system design and management, mobile yellow pages, location-based billing, intelligent transport systems, improved transport systems, and global positioning systems (GPS) [1], [2]. For short-range networks and indoor positioning systems, inventory tracking, intruder detection, tracking of fire-fighters and miners, home automation and patient monitoring applications are examples that employ wireless positioning techniques [3].

One classification criterion for positioning mechanisms is the information that is used in the estimation of the location. In direct positioning, the signals transmitted and received between the nodes are used for calculating the location. On the other hand, in two-step positioning, some parameters related to the position are extracted from the signals first, and then the position is estimated by using those parameters [4]. The timedelay parameter is one of the most widely used ones among these parameters, which is the focus in this study.

A multiple-input multiple-output (MIMO) system uses multiple antennas at the transmitter and the receiver in order to

The authors wish to acknowledge the activity of the Network of Excellence in Wireless COMmunications NEWCOM++ of the European Commission (contract n. 216715) that motivated this work. This work was supported in part by the WiMAGIC project of the EC Seventh Framework Programme (FP7) under Grant agreement no. 215167. provide space diversity [5]. MIMO systems will be used very widely in future communications systems since they provide advantages in terms of quality, reliability, and capacity [5], [6]. MISO systems, which are special cases of MIMO systems, have multiple antennas at the transmitter but have a single antenna at the receiver. In this case, the space diversity can be called as the transmit diversity.

Since positioning is an important application area of wireless systems, it is important to quantify the advantages of space diversity for positioning applications. Although the advantages of space diversity are investigated thoroughly for communications purposes [7] and radar systems [8], [9], [10], there are a few studies in the literature that investigate the effects of space diversity for positioning purposes. For example, [11] studies the space diversity that can be obtained via the use of multiple receive antennas. Mainly, it obtains the theoretical limits, in terms of the Cramer-Rao lower bound (CRLB), on range (equivalently, time-delay) estimation, and proposes a two-step asymptotically optimal range estimator.

Although the effects of receive diversity are studied in [11], no studies have quantified the effects of transmit diversity for time-delay estimation and investigated optimal estimation. In this paper, the time-delay estimation problem in MISO systems is analyzed. First, the signal model is constructed in Section II. Then, the maximum likelihood (ML) time-delay estimator is provided and a theoretical analysis is performed in Section III by deriving the CRLB on time-delay estimation in a MISO system. In Section IV, a genetic global optimization algorithm, called differential evolution (DE), is used to estimate the timedelay parameter from the ML estimator formulation. In that way, the theoretical bound can be achieved at high signal-tonoise ratios (SNRs) with a significantly lower computational complexity than the direct solution of the ML estimator via an exhaustive search. In DE, a number of parameter vectors are generated and updated at each generation in order to reach the global optimum [12], and these vectors encounter mutation, crossover, and selection steps at each generation [13]. Finally, simulation results are presented and concluding remarks are made.

\section{Signal Model}

Consider a MISO system with $M$ antennas at the transmitter and a single antenna at the receiver, as shown in Fig. 1. 


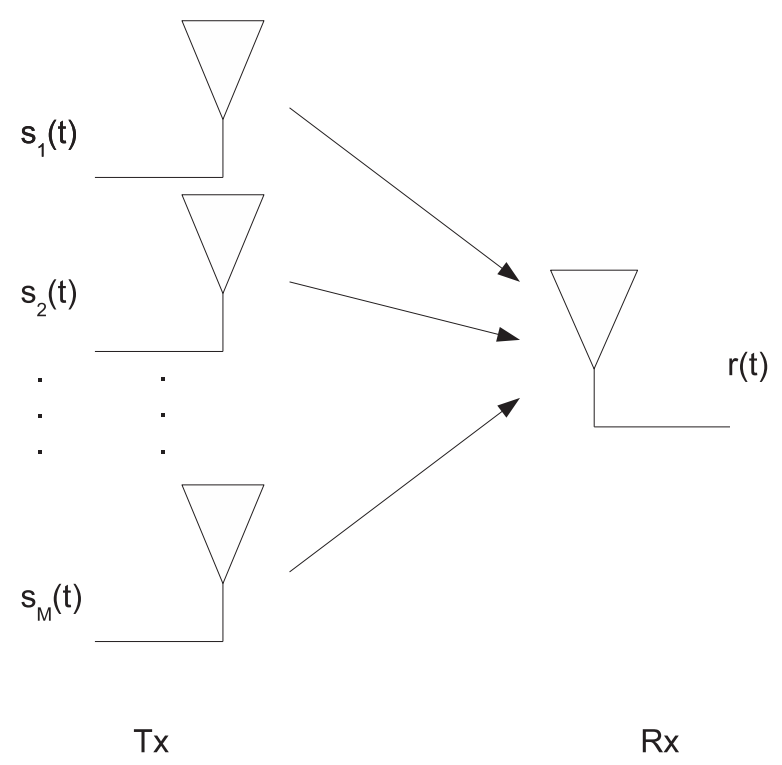

Fig. 1. A MISO system with $M$ transmit antennas.

The baseband received signal at the receiver antenna can be modeled as follows:

$$
r(t)=\sum_{i=1}^{M} \alpha_{i} s_{i}(t-\tau)+n(t),
$$

where $\alpha_{i}=a_{i} e^{j \phi_{i}}$ is the channel coefficient of the $i$ th transmitter branch, $\tau$ is the time-delay, $s_{i}(t)$ is the baseband representation of the transmitted signal from the $i$ th transmit antenna, and $n(t)$ denotes the complex additive white Gaussian noise with independent components each having zero mean and spectral density $\sigma^{2}$.

For the signal model in (1), it is assumed that the signals $s_{1}(t), \ldots, s_{M}(t)$ are narrowband signals, and the differences in the time-delays of the signals coming from different antennas are very small compared to the duration of the signals. Hence, the time-delay parameter can be modeled by a single parameter $\tau$ as in (1). In addition, the transmit antennas are assumed to be separated sufficiently (on the order of signal wavelength) in such a way that the channel coefficients for signals coming from different antennas are independent, which is the main source of transmit diversity in the system.

\section{THEORETICAL LIMITS}

The time-delay estimation problem involves the joint estimation of $\tau$ and the other unknown parameters of the received signal in (1). The unknown signal parameters are denoted by vector $\boldsymbol{\lambda}$ that is expressed as

$$
\boldsymbol{\lambda}=\left[\begin{array}{lllllll}
\tau & a_{1} & \cdots & a_{M} & \phi_{1} & \cdots & \phi_{M}
\end{array}\right] .
$$

If the received signal is observed over the time interval $[0, T]$, then the log-likelihood function for $\boldsymbol{\lambda}$ can be expressed as [14]

$$
\Lambda(\boldsymbol{\lambda})=k-\frac{1}{2 \sigma^{2}} \int_{0}^{T}\left|r(t)-\sum_{i=1}^{M} \alpha_{i} s_{i}(t-\tau)\right|^{2} d t,
$$

where $k$ is a term independent of $\boldsymbol{\lambda}$.

After some manipulation, we obtain the Fisher information matrix (FIM) [14] from (3) as

$$
\mathbf{I}=\left[\begin{array}{lll}
\mathbf{I}_{\tau \tau} & \mathbf{I}_{\tau a} & \mathbf{I}_{\tau \phi} \\
\mathbf{I}_{\tau a}^{T} & \mathbf{I}_{a a} & \mathbf{I}_{a \phi} \\
\mathbf{I}_{\tau \phi}^{T} & \mathbf{I}_{a \phi}^{T} & \mathbf{I}_{\phi \phi}
\end{array}\right]
$$

where the submatrices of the FIM are given by ${ }^{1}$

$$
\begin{aligned}
\mathrm{I}_{\tau \tau} & =\mathrm{E}\left\{\left(\frac{\partial \Lambda(\boldsymbol{\lambda})}{\partial \tau}\right)^{2}\right\} \\
& =\frac{1}{\sigma^{2}} \int_{0}^{T}\left|\sum_{i=1}^{M} \alpha_{i} s_{i}^{\prime}(t-\tau)\right|^{2} d t=\frac{\hat{E}_{s}}{\sigma^{2}},
\end{aligned}
$$

$$
\begin{aligned}
{\left[\mathbf{I}_{a a}\right]_{k k} } & =\mathrm{E}\left\{\left(\frac{\partial \Lambda(\boldsymbol{\lambda})}{\partial a_{k}}\right)^{2}\right\} \\
& =\frac{1}{\sigma^{2}} \int_{0}^{T}\left|s_{k}(t-\tau)\right|^{2} d t=\frac{E_{k}}{\sigma^{2}}, \\
{\left[\mathbf{I}_{a a}\right]_{k n} } & =\mathrm{E}\left\{\frac{\partial \Lambda(\boldsymbol{\lambda})}{\partial a_{k}} \frac{\partial \Lambda(\boldsymbol{\lambda})}{\partial a_{n}}\right\}=\frac{P_{k, n}}{\sigma^{2}}, \text { if } k \neq n, \\
{\left[\mathbf{I}_{\phi \phi}\right]_{k k} } & =\mathrm{E}\left\{\left(\frac{\partial \Lambda(\boldsymbol{\lambda})}{\partial \phi_{k}}\right)^{2}\right\} \\
& =\frac{a_{k}^{2}}{\sigma^{2}} \int_{0}^{T}\left|s_{k}(t-\tau)\right|^{2} d t=\frac{a_{k}^{2} E_{k}}{\sigma^{2}}, \\
{\left[\mathbf{I}_{\phi \phi}\right]_{k n} } & =\mathrm{E}\left\{\frac{\partial \Lambda(\boldsymbol{\lambda})}{\partial \phi_{k}} \frac{\partial \Lambda(\boldsymbol{\lambda})}{\partial \phi_{n}}\right\}=\frac{R_{k, n}}{\sigma^{2}}, \text { if } k \neq n \\
{\left[\mathbf{I}_{\tau \phi}\right]_{k} } & =\mathrm{E}\left\{\frac{\partial \Lambda(\boldsymbol{\lambda})}{\partial \tau} \frac{\partial \Lambda(\boldsymbol{\lambda})}{\partial a_{k}}\right\}=\frac{-F_{k}}{\sigma^{2}}, \\
{\left[\mathbf{I}_{a \phi}\right]_{k k} } & =\mathrm{E}\left\{\frac{\partial \Lambda(\boldsymbol{\lambda})}{\partial \Lambda} \frac{\partial \Lambda(\boldsymbol{\lambda})}{\partial \phi_{k}}\right\}=\frac{-G_{k}}{\sigma^{2}}, \\
{\left[\mathbf{I}_{a \phi}\right]_{k n} } & =\mathrm{E}\left\{\frac{\partial \Lambda(\boldsymbol{\lambda})}{\partial \Lambda(\boldsymbol{\lambda})} \frac{\partial \Lambda(\boldsymbol{\lambda})}{\partial \phi_{k}}\right\}=0, \frac{-H_{k, n}}{\sigma^{2}}, \text { if } k \neq n
\end{aligned}
$$

with

$$
E_{k} \triangleq \int_{0}^{T}\left|s_{k}(t-\tau)\right|^{2} d t
$$

$$
\hat{E}_{s} \triangleq \int_{0}^{T}\left|\sum_{i=1}^{M} \alpha_{i} s_{i}^{\prime}(t-\tau)\right|^{2} d t
$$

$P_{k, n} \triangleq \int_{0}^{T} R e\left\{s_{k}^{*}(t-\tau) s_{n}(t-\tau) e^{j\left(\phi_{n}-\phi_{k}\right)}\right\} d t$

\footnotetext{
${ }^{1}[\mathbf{X}]_{k n}$ denotes the element of matrix $\mathbf{X}$ in row $k$ and column $n$.
} 


$$
\begin{gathered}
R_{k, n} \triangleq \int_{0}^{T} \operatorname{Re}\left\{\alpha_{k}^{*} \alpha_{n} s_{k}^{*}(t-\tau) s_{n}(t-\tau)\right\} d t \\
F_{k} \triangleq \int_{0}^{T} \operatorname{Re}\left\{e^{-j \phi_{k}} s_{k}^{*}(t-\tau) \sum_{i=1}^{M} \alpha_{i} s_{i}^{\prime}(t-\tau)\right\} d t \\
G_{k} \triangleq \int_{0}^{T} \operatorname{Im}\left\{\alpha_{k}^{*} s_{k}^{*}(t-\tau) \sum_{i=1}^{M} \alpha_{i} s_{i}^{\prime}(t-\tau)\right\} d t
\end{gathered}
$$

and

$$
H_{k, n} \triangleq \int_{0}^{T} \operatorname{Im}\left\{a_{n} e^{j\left(\phi_{n}-\phi_{k}\right)} s_{k}^{*}(t-\tau) s_{n}(t-\tau)\right\} d t .
$$

Since the CRLB for the time-delay parameter is given by the first element of the inverse of the FIM, namely, $\left[\mathbf{I}^{-1}\right]_{11}$, the expressions above should be used to obtain the result numerically in general. However, under certain assumptions, closed-form CRLB expressions can be obtained as shown below.

Assumption 1: Assume $\int_{0}^{T} s_{k}^{*}(t) s_{n}^{\prime}(t) d t=0$ for $\forall k \neq n$.

Under this assumption, $\mathbf{I}_{\tau a}$ and $\mathbf{I}_{\tau \phi}$ become $\mathbf{0}$. Then, the CRLB for $\tau$ becomes

$$
\mathrm{CRLB}=\left[\mathbf{I}^{-1}\right]_{11}=\frac{\sigma^{2}}{\hat{E}_{s}} .
$$

Assumption 2: Assume $\int_{0}^{T} s_{k}(t) s_{n}^{*}(t) d t=0$ for $\forall k \neq n$ (orthogonality condition).

Under this assumption, (7), (9), and (13) become zero. For an arbitrary matrix $\mathbf{E}=\left[\begin{array}{ll}\mathbf{A} & \mathbf{B} \\ \mathbf{C} & \mathbf{D}\end{array}\right],\left[\mathbf{E}^{-1}\right]_{M \times M}=(\mathbf{A}$ $\left.\mathbf{B D}^{-1} \mathbf{C}\right)^{-1}$, where $\mathbf{A}$ is an $M$-by- $M$ matrix. If applied to the FIM provided in (4) as

$$
\begin{aligned}
A & =\mathrm{I}_{\tau \tau}, \\
\mathbf{B} & =\left[\begin{array}{ll}
\mathbf{I}_{\tau a} & \mathbf{I}_{\tau \phi}
\end{array}\right], \\
\mathbf{C} & =\mathbf{B}^{T}, \\
\mathbf{D} & =\left[\begin{array}{ll}
\mathbf{I}_{a a} & \mathbf{I}_{a \phi} \\
\mathbf{I}_{a \phi}^{T} & \mathbf{I}_{\phi \phi}
\end{array}\right],
\end{aligned}
$$

then the CRLB; that is, $\left[\mathbf{I}^{-1}\right]_{11}$, can be calculated as follows:

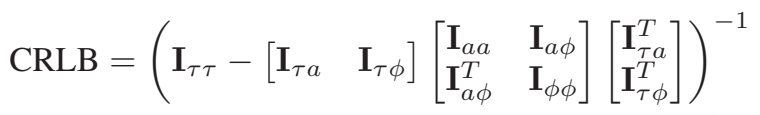

$$
\begin{aligned}
& =\left(\frac{\hat{E}_{s}}{\sigma^{2}}-\frac{1}{\sigma^{2}} \sum_{k=1}^{M} \frac{F_{k}^{2}}{E_{k}}-\frac{1}{\sigma^{2}} \sum_{k=1}^{M} \frac{G_{k}^{2}}{E_{k} a_{k}^{2}}\right)^{-1} \\
& =\frac{\sigma^{2}}{\hat{E}_{s}-\sum_{k=1}^{M}\left(\frac{F_{k}^{2} a_{k}^{2}+G_{k}^{2}}{E_{k} a_{k}^{2}}\right)} \text {. }
\end{aligned}
$$

The CRLB must be minimized in order to maximize the time-delay estimation accuracy. From (21) and (26), it is observed that the maximization of $\hat{E}_{s}$ in (15) is critical in order to achieve the minimum CRLB. In order to provide intuition about how space diversity can be achieved in MISO systems, consider the following two cases:

- If $s_{i}(t)=s(t)$ for all $i$, then $\hat{E}_{s}$ will reduce to

$$
\hat{E}_{s}=\left|\sum_{i=1}^{M} \alpha_{i}\right|^{2} \int_{0}^{T}\left|s^{\prime}(t-\tau)\right|^{2} d t=|\alpha|^{2} \hat{E}
$$

where $\alpha=\sum_{i=1}^{M} \alpha_{i}$ and $\hat{E}=\int_{0}^{T}\left|s^{\prime}(t-\tau)\right|^{2} d t$. It is observed that when the signals are selected to be equal to each other, then $\hat{E}_{s}$ can be undesirably small due to fading and result in a large CRLB. In this case, no space diversity is available in the system.

- If $s_{i}^{\prime}(t)$ 's are orthogonal to each other, then

$$
\begin{aligned}
& \hat{E}_{s}=\int_{0}^{T}\left\{\sum_{i=1}^{M} \sum_{j=1}^{M} \alpha_{i} \alpha_{j}^{*} s_{i}^{\prime}(t-\tau) s_{j}^{\prime *}(t-\tau)\right\} d t \\
& =\sum_{i=1}^{M}\left|\alpha_{i}\right|^{2} \int_{0}^{T}\left|s_{i}^{\prime}(t-\tau)\right|^{2} d t=\sum_{i=1}^{M}\left|\alpha_{i}\right|^{2} \hat{E}_{i}
\end{aligned}
$$

where $\hat{E}_{i}=\int_{0}^{T}\left|s_{i}^{\prime}(t-\tau)\right|^{2} d t$. In this case, the signals are selected so that their derivatives are orthogonal. Such a signal design results in a more robust CRLB by utilizing the transmit diversity in the system. Specifically, even if some of the signals are under deep fades, the other signals can still have reasonably large channel coefficients and can provide a reasonably large $\hat{E}_{s}$ value. Hence, the CRLB will stay reasonably low, which means that accurate time-delay estimation can still be possible in those scenarios. Therefore, the space diversity is utilized in that case.

\section{ML Estimation Based On Differential EVOLUTION}

\section{A. ML Estimator}

From (3), the maximum likelihood (ML) estimator for $\boldsymbol{\lambda}$ can be obtained as

$$
\begin{gathered}
\Lambda(\boldsymbol{\lambda})=\arg \max _{\boldsymbol{\lambda}} \int_{0}^{T}\left\{r(t) \sum_{i=1}^{M} \alpha_{i}^{*} s_{i}^{*}(t-\tau)+\right. \\
\left.r^{*}(t) \sum_{i=1}^{M} \alpha_{i} s_{i}(t-\tau)-\sum_{i=1}^{M} \alpha_{i} s_{i}(t-\tau) \sum_{i=1}^{M} \alpha_{i}^{*} s_{i}^{*}(t-\tau)\right\} d t
\end{gathered}
$$

Under certain conditions, the ML estimator achieves the CRLB asymptotically [14]. However, an exhaustive search approach to find the ML solution for this estimation problem introduces tremendous computational overhead in the presence of multiple transmit antennas. Therefore, there exists a need for finding an algorithm that will obtain the ML solution (approximately) with a low computational load. For that purpose, first, the particle swarm optimization (PSO) approach is tested, which is a renown global optimization algorithm [15]. Despite the general success of the algorithm, it occasionally gets trapped in local minima and does not provide similar results on different trials for the time-delay estimation in MISO systems. Such a problem of PSO is also highlighted 
in [16], and it is also mentioned that DE is more efficient and robust than PSO in certain cases.

\section{B. Differential Evolution (DE)}

DE is a global optimization algorithm with simplicity, reliability, and high performance features. It is proposed by Storn and Price [17], and intended especially for usage in continuous optimization [18]. It is similar to the evolutionary algorithms, but in terms of the new candidate set generation and selection scheme, it differs from them. It does not recombine the solutions using probabilistic schemes but uses the differences of the population members [18]. The basic steps of DE are as follows [13], [16], [18]:

- Initialization: For a global optimization problem with $D$ parameters, a population comprised of $N P$ individuals, which are $D$-dimensional vectors, is generated. The individuals are uniformly distributed all over the optimization space. At each generation, the population is updated according to the update rules used in the next steps. (The parameter vectors at generation $G$ are denoted by $x_{i, G}$ for $i=1,2, \ldots, N P$.)

- Mutation: In this step, for each individual (target vector $\left.\left(x_{i, G}\right)\right)$, three more individuals $\left(x_{r 1, G}, x_{r 2, G}, x_{r 3, G}\right)$ are randomly selected from the population so that all of the four individuals are different from each other. Then, a mutant vector $v_{i, G+1}$ is generated using $x_{r 1, G}, x_{r 2, G}$, $x_{r 3, G}$ in the following way:

$$
v_{i, G+1}=x_{r 1, G}+F\left(x_{r 2, G}-x_{r 3, G}\right)
$$

where $F$ is the amplification factor of the differential variation $\left(x_{r 2, G}-x_{r 3, G}\right)$. Since the search is based on the difference between the individuals, at the beginning of the evolution, the search is distributed all over the search space. However, as the evolution continues, the search is concentrated in the neighborhood of the possible solution. As the difference between the individuals decreases, the step-size is automatically adapted to this situation and decreased.

- Crossover: A crossover between the target vector and the mutant vector is done, which means the elements of them are mixed according to the following rule:

$$
u_{j, i, G}=\left\{\begin{array}{ll}
v_{i, G+1}, & \text { if } \operatorname{rand}(0,1)<C R \\
x_{i, G}, & \text { otherwise }
\end{array},\right.
$$

where $C R$ is the crossover probability for each element of $u_{j, i, G}$. If $C R$ is 0 , then no crossover is performed, which means that all of the elements of $u_{j, i, G}$ are taken from the target vector $x_{i, G}$. Conversely, if $C R$ is 1 , then the mutant vector is copied directly to $u_{j, i, G}$.

- Selection: The decision on the new population member is done greedily in DE. If $u_{j, i, G}$ has a better cost function value than $x_{i, G}$, then $u_{j, i, G}$ takes place of $x_{i, G}$ in generation $G+1$. If the reverse is valid, then $x_{i, G}$ retains its place in the next generation.
This is the standard version of DE, which is also known as "DE/rand/1". The general notation for representing the DE variants is "DE/x/y", where " $\mathrm{x}$ " denotes the selection method of the target vector and " $y$ " denotes the number of difference vectors used. In the standard version, the target vector is chosen randomly, hence " $x$ " is "rand". There is only one difference vector $\left(x_{r 2, G}-x_{r 3, G}\right)$ used for improvement of the evolution, hence " $y$ " is " 1 " [13], [18]. There are many variants of DE proposed in the literature, such as "DE/best/1", "DE/cur-to-best/1", "DE/best/2", "DE/rand/2", and "DE/randto-best/2" [19].

There are three parameters of DE as can be observed above. They are the crossover probability $(C R)$, the amplification factor for the differential variation $(F)$, and the population size $(N P)$. Finding the correct settings of these parameters for a given problem may be a difficult and non-intuitive task [18]. Additionally, different problems may have very different parameter settings [20]. According to the No Free Lunch Theorems, if an algorithm performs well in some set of optimization problems, then it will perform badly for the other set of problems which means some other algorithms will perform well for the second set [21]. The reflection of this concept to the parameter setting problem of DE is finding different parameters that work well for different problems.

Although it is hard to find the optimum parameters for an arbitrary problem and general rules for these parameters, there are many studies on the parameter setting problem in DE. For example, in [12], it is suggested to use 10 times the dimensionality as the population size $(N P)$. Increasing the population size will result in a more explorative and slower algorithm. In our trials on DE parameters, it is seen that with population size 50 , which is 10 times the dimensionality of the problem ( 5 dimensions for two transmit antenna), the algorithm is not as successful as in the case of the population size of 100 . Therefore, 100 is preferred in this study. Generally, the recommendation for the $C R$ value is 0.9 [22], [23]. So, we used 0.9 in our study. For the amplification factor $(F)$, there are many recommended values. But, they are generally between 0.5 and 1 [22]. The best performance in this optimization problem is obtained when $F$ is selected as 0.5 .

The stopping criterion for the algorithm is selected as the iteration count. Although it is observed that 100-150 iterations are generally sufficient, to be on the safe side, the iteration count is selected as 200 .

\section{Simulation Results}

In this section, simulations performed by using the DE algorithm studied in Section IV are provided. The performance of DE is compared with the theoretical limit (CRLB) under different simulation scenarios. In the simulations, for $s_{i}(t)$, the modified Hermite pulses (MHPs) are used [24], [25]. $s_{1}(t)$ is the second order MHP and $s_{2}(t)$ is the third order MHP; that is,

$$
s_{1}(t)=\frac{e^{-t^{2} / 4 \beta^{2}}}{\beta^{2}}\left(\frac{t^{2}}{\beta^{2}}-1\right)
$$




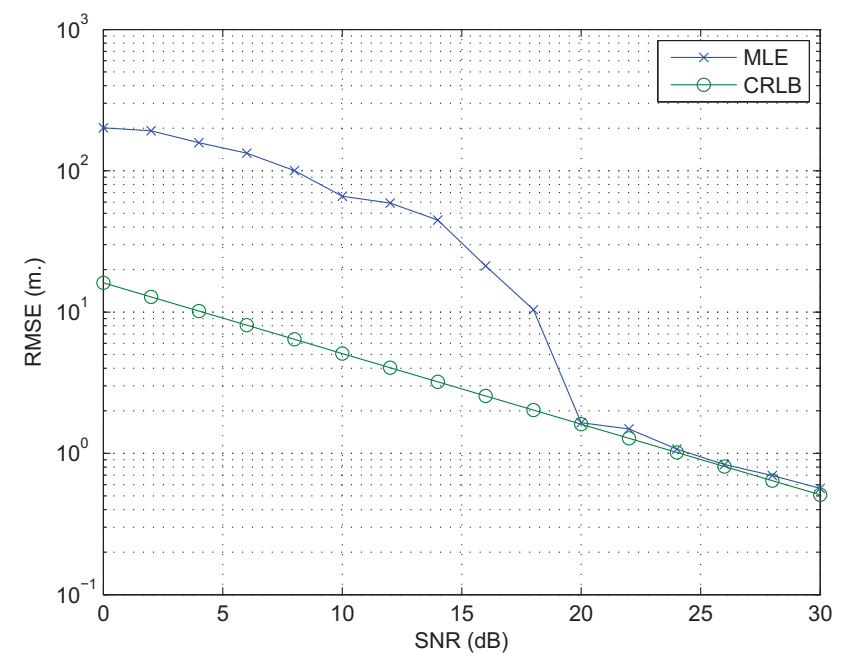

Fig. 2. The RMSE of the MLE and the square-root of the CRLB for the Rayleigh fading channel.

$$
s_{2}(t)=\frac{e^{-t^{2} / 4 \beta^{2}}}{\beta^{4}}\left(\frac{t^{3}}{\beta^{2}}-3 t\right),
$$

where $\beta$ is the parameter used for adjusting the pulse according to a given pulse width $(P W)$. It is selected as $P W / 15$ in the simulations. The MHPs are selected because of their orthogonality property. Since the orthogonality property of the signals satisfy Assumption 2 provided in Section III, the results of the simulations are compared to the square-root of the CRLB (theoretical bound) calculated under this assumption. The derivatives of the MHPs are also approximately orthogonal; hence, the space diversity explained in Section III is present. The ML estimator reaches the theoretical bound under certain conditions. However, it is computationally very complex to obtain the exact ML solution via an exhaustive search compared to the ML solution by using DE. The simulations depict the performance of the DE based ML solution for different channel conditions.

The first simulation is performed under a Rayleigh fading channel condition, and the results are illustrated in Fig. 2. For Rayleigh fading, the amplitudes of the channel coefficients $\left(\alpha_{i}=a_{i} e^{j \theta_{i}}\right)$ can differ significantly. Therefore, the utilization of diversity is very important in this scenario. In the figure, the SNR of the system is defined as SNR $=10 \log _{10}\left(E /\left(2 \sigma^{2}\right)\right)$, where $E$ is the energy of the signal transmitted from one antenna. The channel coefficients $\left(a_{i}\right)$ are Rayleigh distributed random variables with an average power of unity $\left(\mathrm{E}\left\{a_{i}^{2}\right\}=1\right)$ and $\phi_{i}$ 's are distributed as uniform random variables in the interval $[0,2 \pi)$. The time-delay $\tau$ is generated according to uniform distribution over the observation interval. The bandwidths of the signals are set to $B=1 \mathrm{MHz}$. The root mean-squared error (RMSE) is calculated over many channel realizations and this is compared to the square-root of the CRLB.

The second simulation investigates the performance of the

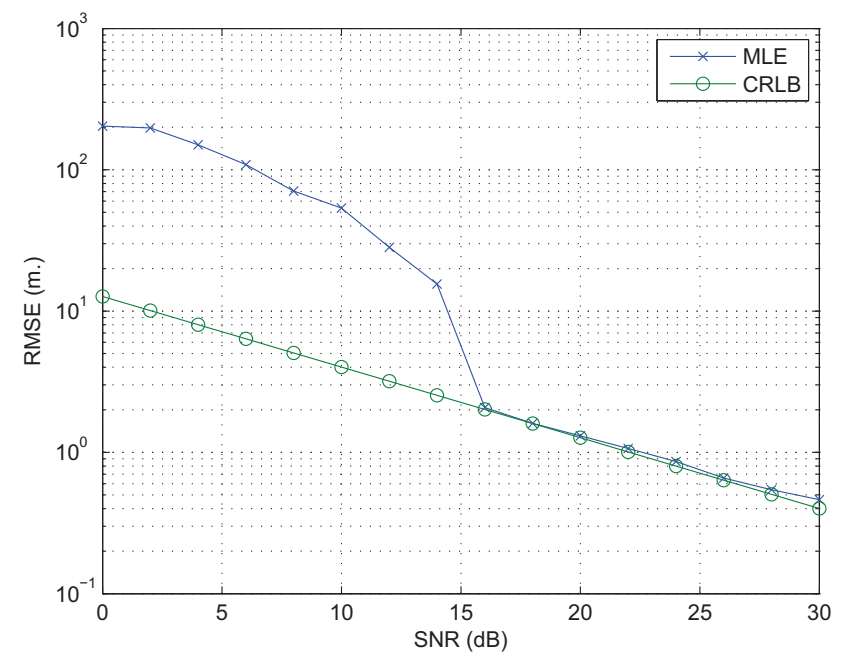

Fig. 3. The RMSE of the MLE and the square-root of the CRLB for the Rician fading channel $(K=5)$.

DE based ML algorithm under Rician fading with a $K$ factor of 5. The SNR definition, the average power of the channel coefficients, and the distribution of $a_{i}$ 's and $\phi_{i}$ 's are the same as in the previous simulations. Fig. 3 illustrates the performance of the algorithm in this scenario. Again, it is observed that the RMSE of the estimator converges to the CRLB at high SNRs, and the space diversity introduced by the transmit antennas is utilized. Also, comparison of Fig. 2 and Fig. 3 reveals that the RMSE of the DE based ML algorithm is lower for the Rician fading channel, which is expected since Rician fading corresponds to a less severe channel condition than Rayleigh fading. In addition, the RMSE of the estimator converges to the CRLB at lower SNRs in the Rician fading scenario.

\section{CONCLUding Remarks}

The CRLB is the theoretical limit that provides the lower bound on the variance of an unbiased estimator. In this paper, the CRLB expressions have been calculated for MISO systems. The ML estimator achieves the CRLB under certain conditions; however, it can have very high computational complexity. To avoid this, a genetic global optimization algorithm has been used to obtain the ML estimate with lower computational complexity. It has been observed that DE can find the ML estimate much more rapidly than the exhaustive search method, and that the parameters of DE are crucial in terms of obtaining the optimum point that maximizes the loglikelihood function. The optimum parameter values have been selected for DE and applied in the algorithm.

In addition, this study has emphasized the importance of space diversity for the time-delay estimation problem in MISO systems. It has been stated that the transmit diversity can be utilized by using signals with orthogonal derivatives.

Finally, the performance of DE has been tested under various channel conditions. It has been observed that the DE 
based ML algorithm converges to the CRLB at lower SNRs in the Rician fading channel than in the Rayleigh fading one. However, in both of the scenarios, the algorithm reaches the CRLB at sufficiently high SNRs by utilizing the transmit diversity, with much lower computational complexity than the ML estimator via an exhaustive search.

\section{REFERENCES}

[1] J. J. Caffery, Wireless Location in CDMA Cellular Radio Systems, Boston Kluwer, 2000

[2] Z. Sahinoglu, S. Gezici, and I. Guvenc, Ultra-wideband Positioning Systems: Theoretical Limits, Ranging Algorithms, and Protocols, New York: Cambridge University Press, 2008.

[3] "IEEE 15-03-0489-03-004a-application-requirement-analysis-031127 v0.4.[Online]", http://www.ieee802.org/15/pub/TG4.html

[4] S. Gezici, "A survey on wireless position estimation," Wireless Personal Communications, vol. 44, no. 3, pp. 263-282, Feb. 2008.

[5] E. Biglieri, A. Constantinides, R. Calderbank, A. Goldsmith, A. Paulraj, and H. V. Poor, MIMO Wireless Communications, Cambridge Univ. Press, 2007.

[6] R. W. Heath and A. Paulraj, "Switching between diversity and multiplexing in MIMO systems," IEEE Transactions on Communications, vol. 53 , issue 6, pp. 962-968, June 2005.

[7] A. Goldsmith, Wireless Communications, Cambridge, UK: Cambridge University Press, 2005.

[8] E. Fishler, A. Haimovich, R. Blum, L. Cimini, D. Chizhik, and R. Valenzuela, "MIMO radar: An idea whose time has come," Proc. IEEE Int. Conf. on Radar, Philadelphia, PA, April 2004.

[9] J. Li and P. Stoica, "MIMO radar - Diversity means superiority," Proc. 14th Adaptive Sensor Array Process. Workshop (ASAP'06), MIT Lincoln Laboratory, Lexington, MA, Jun. 2006.

[10] L. Xu, J. Li, and P. Stoica, "Radar imaging via adaptive MIMO techniques," Proc. 14th European Signal Processing Conf., Florence, Italy, Sept. 2006.

[11] S. Gezici and Z. Sahinoglu, "Ranging in a single-input multiple-output (SIMO) system," IEEE Commun. Lett., vol. 12, no. 3, pp. 197-199, Mar. 2008.

[12] R. Storn and K. Price, "Differential evolution - A simple and efficient heuristic for global optimization over continuous spaces," Journal of Global Optimization, vol. 11, pp. 341-359, 1997.

[13] R. Gämperle, S. D. Müller, and P. Koumoutsakos, "A parameter study for differential evolution," Advances in Intelligent Systems, Fuzzy Systems, Evolutionary Computation, WSEAS Press, pp. 293-298, 2002.

[14] H. V. Poor, An Introduction to Signal Detection and Estimation, 2nd ed., New York: Springer, 1994.

[15] J. Kennedy and R. C. Eberhart, "Particle swarm optimization," Proc. IEEE International Conference on Neural Networks, Piscataway, NJ., pp. 1942-1948, 1995.

[16] J. Vesterstrøm and R. Thomsen, "A comparative study of differential evolution, particle swarm optimization, and evolutionary algorithms on numerical benchmark problems," Congress on Evolutionary Computation (CEC2004), vol. 2, pp. 1980-1987, 2004.

[17] R. Storn and K. Price, "Differential evolution - A simple and efficient adaptive scheme for global optimization over continuous spaces," Tech nical Report, International Computer Science Institute, Berkeley, 1995.

[18] F. Neri and V. Tirronen, "Recent advances in differential evolution: A survey and experimental analysis," Artificial Intelligence Review, vol. 33, no. 1-2, pp. 61-106, 2010

[19] A. K. Qin and P. N. Suganthan, "Self-adaptive differential evolution algorithm for numerical optimization," in Proc. IEEE Congress on Evolutionary Computation, vol. 2, pp. 1785-1791, 2005.

[20] R. Mallipeddi and P. N. Suganthan, "Empirical study on the effect of population size on differential evolution algorithm," in Proc. IEEE Congress on Evolutionary Computation, pp. 3663-3670, 2008.

[21] D. Wolpert and W. Macready, "No free lunch theorems for optimization," IEEE Transactions on Evolutionary Computation, vol. 1, no. 1, pp. 67-82, April 1997.

[22] J. Liu and J. Lampinen, "On setting the control parameter of the differential evolution algorithm," in Proc. 8th International Mendel Conference on Soft Computing, pp. 11-18, 2002.
[23] J. Rökkönen, S. Kukkonen, and K. V. Price, "Real-parameter optimization with differential evolution," in Proc. IEEE International Conference on Evolutionary Computation, vol. 1, pp. 506-513.

[24] H. Harada, K. Ikemoto, and R. Kohno, "Modulation and hopping using modified Hermite pulses for UWB communications," Proc. IEEE Conference of Ultra Wideband Systems and Technologies (UWBST 2004), pp. 336-340, Kyoto, Japan, May 2004.

[25] C. Mitchell and R. Kohno, "High data rate transmissions using orthogonal modified Hermite pulses in UWB Communications," International Conferences on Telecommunications, ICT 2003, vol. 2, pp. 1278-1283, March 2003. 